\section{THU0156 CLINICAL ASSESSMENT VERSUS ULTRASONOGRAPHY IN PATIENTS WITH RHEUMATOID ARTHRITIS TREATED WITH BIOLOGICAL AGENTS - THE IMPACT OF CONCOMITANT FIBROMYALGIA}

M.M. Tamas ${ }^{1}$, L.-J. Ghib ${ }^{1}$, C.I. Bondor ${ }^{2}$, I. Felea ${ }^{3}$, L. Damian ${ }^{3}$, I. Filipescu ${ }^{1}$, A. Mociran ${ }^{3}$, S.-P. Simon ${ }^{1}$, L. Muntean ${ }^{1}$, S. Rednic ${ }^{1}$. ${ }^{1}$ Rheumatology; ${ }^{2}$ Department of Medical Informatics and Biostatistics, "Iuliu Hatieganu" University of Medicine and Pharmacy; ${ }^{3}$ Rheumatology, Emergency County Clinical Hospital, Cluj Napoca, Romania

Background: Concomitant fibromyalgia (FM) may increase subjective components of the disease activity scores (DAS) in rheumatoid arthritis (RA), thus leading to an improper assessment of treatment response. Ultrasonography (US) is currently used in clinical practice as an objective measure of the disease activity. The US7 German score was demonstrated to be sensitive to change, reflecting the therapeutic response in RA patients [1]

Objectives: To evaluate clinical and US parameters in RA patients with or without concomitant FM, undergoing biological therapy.

Methods: RA patients treated with various biological agents who presented in our Department of Rheumatology were consecutively enrolled. Patients underwent clinical and laboratory examinations. US was performed by an experienced sonographer, blinded to clinical evaluation. Synovitis and synovial/tenosynovial vascularity were scored semiquantitatively (grade $0-3$ ) by gray-scale (GS) and power Doppler (PD) US. Tenosynovitis (TS) and erosions were scored for presence [1]

Patients were divided in two groups according to the difference between tender joint count (TJC) and swollen joint count (SJC): $>7$ RA-FM group, $<7$ RA-nonFM group, representing the "joint count" criteria for FM, after the study of Pollard et al. [2].

Results: Thirty-nine patients were included, $77 \%$ women, mean age $55.2 \pm 11.3$ years, mean disease duration $15.25 \pm 9.4$ years. Nine out of $39(23 \%)$ patients were classified as having associated FM. Disease duration and treatment were comparable between groups.

Significantly higher values for TJC, patient global assessment (PGA), DAS 28 were found in the RA-FM group, with no differences for SJC or inflammatory markers (ESR, CRP). GS and PD-US7 scores were similar between groups (Table 1)

Table 1. Clinical and US findings in RA-nonFM and RA-FM groups

\begin{tabular}{lccc}
\hline & RA-nonFM $(\mathrm{n}=30)$ & RA-FM $(\mathrm{n}=9)$ & $P$ value \\
\hline PGA $(\mathrm{mm})$ & $41.33(18.28)$ & $58.88(17.63)$ & $<0.001$ \\
TJC & $3(0-10)$ & $12(9-17)$ & $<0.001$ \\
SJC & $2(0-7)$ & $1(0-6)$ & 0.54 \\
ESR $(\mathrm{mm} / \mathrm{h})$ & $22.26(18.42)$ & $27.33(16.87)$ & 0.21 \\
CRP $(\mathrm{mg} / \mathrm{l})$ & $8.46(17.84)$ & $8.67(9.11)$ & 0.66 \\
DAS28 CRP & $3.33(0.98)$ & $5.13(1.18)$ & 0.015 \\
GS Synovitis US7 score & $3.80(4.37)$ & $2.67(1.66)$ & 0.96 \\
PD Synovitis US7 score & $1.67(2.90)$ & $1.22(1.20)$ & 0.59 \\
Erosions US7 score & $3.77(2.75)$ & $4.89(2.85)$ & 0.29 \\
GS TS US7 score & $0.30(0.84)$ & $0.44(0.73)$ & 0.31 \\
PD TS US7 score & $0.20(1.10)$ & $0.33(0.71)$ & 0.08 \\
\hline
\end{tabular}

In the RA non-FM, but not in the RA-FM group, GS and PD-US7 correlated with SJC ( $r=0.44, p=0.015$ - GS synovitis, $r=0.47, p=0.008$ - PD synovitis; $r=0.57, p=0.001-$ GS TS; $r=0.46, p=0.011-$ PD TS) and PD synovitis negatively correlated with the "joint count" criteria $(r=-0.44, p=0.015)$.

Conclusions: Concomitant FM in RA patients undergoing biological therapy lead to higher DAS28 scores, but not to synovial inflammation on US. US-PD correlates with clinically detected synovitis in the non-FM group. US is expected to modify treatment decision and to prevent RA mistreatment especially in RA-FM patients. References:

[1] Backhaus M, et al. Evaluation of a novel 7-joint ultrasound score in daily rheumatologic practice: a pilot project. Arthritis Rheum. 2009;61(9):1194-201.

[2] Pollard LC, et al. Fibromyalgic rheumatoid arthritis and disease assessment. Rheumatology (Oxford). 2010;49(5):924-928.

Disclosure of Interest: None declared

DOI: 10.1136/annrheumdis-2017-eular.3971

\section{THU0157 RELATIONSHIP OF MATRIX METALLOPROTEASE 3 TITER AND SARCOPENIA IN PATIENTS WITH RHEUMATOID ARTHRITIS: DATA FROM THE CHIKARA STUDY}

M. Tada, Y. Yamada, K. Mandai, N. Hidaka. Orthopaedic Surgery, Osaka City General Hospital, Osaka, Japan

Background: Patients with rheumatoid arthritis (RA) show a lower muscle mass and higher prevalence of sarcopenia than healthy individuals. A prospective observational study (CHIKARA study, registration number: UMIN000023744) was started to clarify the influence of changes in disease activity for sarcopenia.

Objectives: We investigated the relationship between sarcopenia and disease activity at baseline in patients with RA.

Methods: We analyzed baseline data from the CHIKARA study (Correlation research of sarcopenia, skeletal muscle and disease activity in rheumatoid arthritis). Sarcopenia was diagnosed using the criteria of the Asia Working Group on Sarcopenia ${ }^{2}$. Muscle mass, body fat mass, total body water, bone mass, and basal metabolic rate were measured using a body composition analyzer (MC 780A; TANITA, Tokyo, Japan). We investigated correlations between sarcopenia and disease activity (DAS28-ESR, SDAI, physical function (HAQ), and laboratory data using uni- and multivariate analyses.

Results: Participants comprised 100 patients with RA (females, 78\%; mean age 66.1 years). Mean disease duration was 5.5 years, DAS28-ESR was 3.55 , and the percentage of subjects with sarcopenia was $28 \%$. Table 1 shows risk factors for sarcopenia. Sarcopenia correlated with weight, body mass index (BMI), body fat mass, muscle mass, basal metabolic rate, Steinbrocker stage, CRP bone mass, and matrix metalloprotease (MMP)-3 on univariate analysis. Glucocorticoid dosage, rheumatoid factor, and anti-CCP antibody showed no correlation with sarcopenia. BMI, body fat mass, and MMP-3 were identified as independent risk factors on multivariate analysis. MMP-3 over $90.7 \mathrm{ng} / \mathrm{ml}$ was a risk factor for sarcopenia by ROC curve analysis (odds ratio, 3.09; $p=0.023$ ).

Table 1. Risk factors for sarcopenia in patients with RA

\begin{tabular}{lcccccc}
\hline & \multicolumn{2}{c}{ Univariate } & & \multicolumn{3}{c}{ Multivariate } \\
\cline { 2 - 3 } \cline { 5 - 6 } & $\mathrm{R}$ & $P$ & & Odds ratio & $95 \% \mathrm{Cl}$ & $P$ \\
\hline Weight & -0.421 & $<0.001$ & & - & - & - \\
BMI & -0.490 & $<0.001$ & & 0.307 & $0.185-0.509$ & $<0.001$ \\
Body fat mass & -0.219 & 0.002 & & 1.318 & $1.124-1.546$ & 0.001 \\
Muscle mass & -0.325 & 0.001 & & - & - & - \\
Basal metabolic rate & -0.419 & $<0.001$ & & - & - & - \\
Steinbrocker stage & 0.206 & 0.039 & & - & - & - \\
CRP & 0.201 & 0.045 & & - & - & - \\
Bone mass & -0.374 & $<0.001$ & & - & - & - \\
MMP-3 & 0.238 & 0.017 & & 1.012 & $1.003-0.021$ & 0.01 \\
\hline
\end{tabular}

Conclusions: The percentage of sarcopenia was $28 \%$ in patients with RA. Low $\mathrm{BMI}$, high body fat mass, and high MMP-3 represented independent risk factors for sarcopenia. A relationship between MMP-3 and sarcopenia was indicated by this study.

References:

[1] Inui K., Koike T., Tada M., et al. Sarcopenia is apparent in patients with rheumatoid arthritis, especially those with biologics -TOMORROW studyEULAR 2015 abstract (AB0359)

[2] Chen Lk., Liu LK., Assantachai P., et al. Sarcopenia in Asia: consensus report of the Asian Working Group for Sarcopenia. J Am Med Dir Assoc. 2014; 15 : 95-101.

Disclosure of Interest: None declared

DOI: 10.1136/annrheumdis-2017-eular.2334

\section{THU0158 IMMUNE RESPONSE EFFICIENCY AFTER VACCINATION IN RA AND SPA PATIENTS TREATED WITH BIOLOGICS AND IMMUNOSUPPRESSIVE AGENTS: A SYSTEMATIC LITERATURE REVIEW}

M. Tissier ${ }^{1}$, A. Molto ${ }^{1}$, S. Kolta ${ }^{1}$, O. Fogel ${ }^{1}$, M. Dougados ${ }^{1}$, J. Morel $^{2}$, C. Miceli-Richard ${ }^{1}$. ${ }^{1}$ Hopital Cochin Paris, Paris; ${ }^{2} \mathrm{CHU}$ Montpellier, Montpellier, France

Background: One of the most effective strategies to prevent infections is vaccination, especially in patients treated with biologics and immunosuppressive (IS) agents. Nevertheless, the effectiveness of the resulting immune response in these patients has been questioned.

Objectives: To perform a systematic literature review aiming to assess evidence available regarding immune response efficiency (IRE) and the ideal schedule for vaccination in RA and SpA patients treated with Methotrexate (MTX), TNF inhibitors (TNFi), anti-CD20 (rituximab, RTX), anti-CTLA4 (abatacept, ABA) or anti-IL6 (tocilizumab, TCZ).

Methods: A systematic literature review was conducted by searching in PubMed all studies with the MeSH terms "[Rheumatoïd Arthritis" OR "Spondyloarthritis"] AND ["vaccination" OR "vaccines"] AND ["Methotrexate" OR "Abatacept" OR "Tocilizumab" OR "Rituximab" OR "Adalimumab" OR "Certolizumab" OR "Etanercept" OR "Golimumab" OR "Infliximab"], with no limitation regarding time of publication. Only studies evaluating the IRE were included. Case reports, general reviews and meta-analysis were excluded.

Results: After exclusion criteria, 35 studies (out of 60 studies retrieved) assessing IRE in RA or SpA patients were selected, under MTX $(n=35)$, TNFi $(n=18)$, RTX $(n=8)$, ABA $(n=4)$ or TCZ $(n=5)$. The studied vaccines were mostly the trivalent seasonal Influenza $(n=20)$, the anti-pneumococcal vaccine $(n=16)$, and few studies regarding the tetanus toxoid vaccine (TTV) $(n=2)$, Hepatitis A vaccine $(n=1)$ and accidental revaccination against yellow fever $(n=2)$. Most studies $(32 / 35)$ evaluated the IRE using the antibody (Ab) titer. When studying the anti-pneumococcal and influenza vaccination, the primary outcome was mainly the seroresponse 3 to 6 weeks after vaccination (i.e. Ab ratio post/pre-vaccine) but some studies (15/35) also assessed the seroprotection rate (i.e. patients with an effective titer of protective $A b)$, and some $(3 / 35)$ the effectiveness of seroresponse using the opsonization index rate.

Regarding the anti-pneumococcal and influenza vaccination, MTX, RTX, and ABA were reported to impair the immune response; neither TNFi nor TCZ were shown to decrease seroresponse. 
Regarding TTV, one study showed persistent seroresponse in patients treated with TNFi; a second study showed no difference between MTX and RTX in response to T-cell dependant protein Ag TTV.

Two studies reported no significant difference in efficiency or tolerance in patients accidentally revaccinated against yellow fever under TNFi.

Conclusions: These observations highly suggest that an effective vaccination for patients treated with MTX, RTX, and ABA should necessitate a therapeutic window or a scheduled treatment spacing, in order to offer the best protection against Influenza and Pneumococcal infection. This might not be necessary in patients under TNFi or TCZ; nevertheless, further studies are necessary to optimize the vaccination modalities.

Disclosure of Interest: None declared

DOI: 10.1136/annrheumdis-2017-eular.1396

\section{THU0159 RHEUMATOID ARTHRITIS MAY NOT INFLUENCE EATING BEHAVIOURS CHARACTERISTICS}

N. Bulut ${ }^{1}$, M.E. Tezcan ${ }^{2} .{ }^{1}$ Family Medicine; ${ }^{2}$ Rheumatology, Kartal Research and Education Hospital, Istanbul, Turkey

Background: Patients with rheumatoid arthritis (RA) have an increased risk of obesity and cardiovascular disease compared with the general population (1). Eating behaviours may play an important role in obesity and cardiovascular disease.

Objectives: We hypothesised that RA patients have impaired eating behaviours that may have a role in obesity. For evaluating this hypothesis, we compared eating behaviours of patients with RA, osteoarthritis $(\mathrm{OA})$ and healthy controls. Methods: One hundred and fifty-seven RA patients (M/F: 23/134) who fulfilled the 2010 American College of Rheumatology (ACR) RA classification criteria, 31 hand OA patients (M/F: 1/30) who fulfilled 1990 ACR hand OA criteria and 60 healthy controls ( $M / F: 9 / 51$ ) were enrolled to the study who applied to Kartal Dr. Lutfi Kirdar Training and Research Hospital Rheumatology outpatient clinic. Eating behaviours was assessed by the Three-Factor Eating Questionnaire (TFEQ).Demographic data, smoking status, co-morbidities, anthropometric measurements, VAS pain score were analyzed.

Results: There were no differences between three groups in demographic features and anthropometric measurements (table1). Moreover, there were no differences between three groups in cognitive restraint and emotional eating scores.Although, healthy controls had significantly higher uncontrolled eating scores than the RA group $(p<0.05)$, uncontrolled eating scores of all three groups were lower than Turkish people average scores (2) (table2).

Table 1. Demographic and Anthropometric features of study groups

\begin{tabular}{|c|c|c|c|c|}
\hline & RA $(n=157)$ & $O A(n=31)$ & Healthy Controls $(n=60)$ & $p$ \\
\hline Age & $51.00(40.00-57.50)$ & $50.00(46.75-62.00)$ & $48.00(39.25-53.75)$ & 0.06 \\
\hline Gender (M/F) & 3/134 (14.6/85.4) & $1 / 30(3.2 / 96.8)$ & 9/51 (15.0/85.0) & 0.21 \\
\hline Education (year) & $5.00(5.00-10.00)$ & $5 . .00(5.00-11.00)$ & $5.00(5.00-11.00)$ & 0.42 \\
\hline Smoking $((\%)$ & 24.2 & 23.3 & 20.7 & 0.86 \\
\hline Co-morbid & 55.8 & 64.5 & 49.2 & 0.37 \\
\hline Lenght $(\mathrm{cm})$ & $\begin{array}{c}160.00 \\
(155.00-165.00)\end{array}$ & $\begin{array}{c}157.00 \\
(155.00-160.00)\end{array}$ & $\begin{array}{c}160.50 \\
(157.00-166.75)\end{array}$ & 0.06 \\
\hline & 75.00 & 77.00( & & 0.73 \\
\hline BMI ( & 29.15 & $31.100(27$ & $27.550(23.87-32.77)$ & 0.15 \\
\hline VAS pain $(0-100)$ & $10.00(0.00-30.00)$ & $10.00(10.00-30.00)$ & $\mathrm{N} / \mathrm{A}$ & 0.07 \\
\hline \multicolumn{5}{|c|}{ RA, Rheumatoid Arthritis; OA, Osteoarthritis; BMI, Body mass index; VAS, Visual analog score. } \\
\hline \multicolumn{5}{|c|}{ Table 2. Eating behaviours of study groups } \\
\hline & $\mathrm{RA}(\mathrm{n}=157)$ & $O A(n=31)$ & Healthy Controls $(n=60)$ & $p$ \\
\hline $\begin{array}{l}\text { Uncontrolled eating } \\
\text { scores }\end{array}$ & $10.00(8.00-12.00)$ & $10.00(8.00-13.00)$ & $11.00(9.00-14.00)$ & 0.047 \\
\hline Cognitive restraint & & & & \\
\hline scores & $16.00(13.00-18.00)$ & $16.00(14.00-19.00)$ & $14.00(11.25-18.00)$ & 0.075 \\
\hline motional eating & & & & \\
\hline scores & $5.00(3.00-8.00)$ & $5.00(3.75-11.00)$ & $4.00(3.00-7.00)$ & 0.066 \\
\hline
\end{tabular}

Conclusions: This was the first study using the TFEQ in patients with RA. In our study, we found that disease features of RA may had no effect on eating behaviors.

References:

[1] Kitas GD, Erb N. Tackling ischemic heart disease in rheumatoid arthritis. Rheumatology (Oxford). 2003;42(5):607-13.

[2] Kıraç D, Kaspar EÇ, Avcılar T, Çakır ÖK, Ulucan K, Kurtel H, ve ark. Obeziteyle ilişkili beslenme alışkanlıklarının araştııılmasında yeni bir yöntem "Üç Faktörlü Beslenme Anketi”. MÜSBED. 2015;5(3):162-9.

Acknowledgements: None.

Disclosure of Interest: None declared

DOI: 10.1136/annrheumdis-2017-eular.6596

\section{THU0160 DISEASE MODIFYING ANTIRHEUMATIC DRUGS IN RHEUMATOID ARTHRITIS WITH INTERSTITIAL LUNG DISEASE: A PROSPECTIVE STUDY}

N. Mena-Vazquez ${ }^{1}$, C. Gómez-Cano ${ }^{2}$, C. Aguilar-Hurtado ${ }^{3}$ M. Rojas-Giménez ${ }^{1}$, S. Manrique-Arija ${ }^{1}$, C. Domic ${ }^{1}$, C. Fuego-Valera ${ }^{1}$ I. Ureña-Garnica ${ }^{1}$, F.G. Jiménez-Núñez ${ }^{1}$, G. Diaz-Cordoves ${ }^{1}$,

M.V. Irigoyen-Oyarzábal ${ }^{1}, M$. Padín-Martín ${ }^{3}$, A. Fernández-Nebro ${ }^{1}$.

${ }^{1}$ Department of Rheumatology at the University Regional Hospital of Malaga

(HRUM). Institute for Biomedical Research in Malaga (IBIMA). Malaga

University., Malaga; ${ }^{2}$ Department of Rheumatology Hospital Valme, Sevilla;

${ }^{3}$ Department of Radiology, Malaga, Spain

Objectives: To describe the evolution of interstitial lung disease (ILD) in RA patients treated with disease modifying antirheumatic drugs (DMARDs) for 1 year in real clinical practice conditions.

Methods: Design: Prospective observational case-series. Patients: Patients with RA (ACR/EULAR 2010 criteria) and ILD (American Thoracic Society/European Respiratory criteria) from two centres (Regional Hospital of Málaga and Valme Hospital of Sevilla) were included. Protocol: All patients with RA and ILD who visited outpatient clinic from January to December 2015. They were reviewed according to a predetermined protocol for systematic data collection. Resolution Computed Tomography (HRCT),Pulmonary function test (PFT) and echocardiogram were requested for all patients. This visit was marked as v0 (index date). At 12 months (v12) the joint assessment (DAS28), echocardiogram, PTF and HRCT were again evaluated. HRCT's were assessed by the same radiologist with expertise in chest radiology. Outcomes: At v12:(1)improvement (ie improvement in $\mathrm{FVC} \geq 10 \%$ or $\mathrm{DLCO} \geq 15 \%$ and no radiological progression),(2)non-progression (stabilization or improvement in $\mathrm{FVC} \leq 10 \%$ or $\mathrm{DLCO}<15 \%$ and no radiological progression), (3)progression (worsening of FVC $>10 \%$ or DLCO $>15 \%$ and radiological progression), or (4) death due to ILD. Variables: Description of ILD type (Nonspecific interstitial pneumonia/Usual interstitial pneumonia) and lung function by PTF, HRCT.Presence of PTH by echocardiogram and dyspnoea.Collection of adverse events. Statistical analysis: Descriptive analysis and Wilcoxon or T test between the v0 and v12. One factor ANOVA between SDMARD,bDMARD and combination therapy groups.

Results: The main characteristics at V0 of the patients $(n=22)$ are shown in the table. Seven patients $(31.8 \%)$ received a sDMARDs with a bDMARDs; 12 patients $(54.5 \%)$ in monotherapy with sDMARD, with MTX being the most frequent $(34.7 \%) ; 3(13.6 \%)$ in monotherapy with bDMARDs (Table 1). Three patients (13.6\%) had improvement (1 with MTX, 1 with RTX and 1 with HCX + RTX), 15 patients $(68.2 \%)$ remained stable (4 with MTX, 3 with LFN, 1 with HCQ, 1 AZA, $1 \mathrm{ABT}, 1 \mathrm{ABT}+\mathrm{SSZ}, 2 \mathrm{MTX}+\mathrm{ETN}, 1 \mathrm{HCQ}+\mathrm{RTX}$, and $1 \mathrm{HCQ}+\mathrm{ADA})$; and 3 $(13.0 \%)$ got worse of ILD (1 with MTX developed unknown lung mass, 1 with LFN and 1 with LFN + IFX). One patient died during follow-up due to respiratory infection (under treatment with RTX). No patient developed PPH. We did not find significant differences between v0 DAS28 and at 12 months $(2.55[0.75]$ vs 2.42 [1.22], $p=0.567$ ) or in HAQ 1.15 [0.93] vs 1.25 [0.78], $p=0.450$ ). There were no significant differences in PTF, HRCT or DAS28 at v12 between SDMARD, bDMARD and combination therapy groups. Four patients $(18.2 \%)$ had adverse effects: 2 respiratory infections, 1 oral herpes simplex and 1 tooth infection.

\begin{tabular}{|c|c|}
\hline VARIABLES & Patients \\
\hline Sex (Female), $n(\%)$ & $12(54.5)$ \\
\hline Age (years), mean (DE) & $69.4(7.0)$ \\
\hline Smoker, n (\%) & $4(18.2)$ \\
\hline Body mass index (BMI), mean (SD & $28.9(6.0)$ \\
\hline Disease duration (months), mean (SD) & $211.9(131.5)$ \\
\hline ILD duration (months), mean (SD) & $64.5(47.1)$ \\
\hline Rheumatoid factor, $\mathrm{n}(\%)$ & $21(95.5)$ \\
\hline Anti-cyclic citrullinated peptide, $n(\%)$ & $21(95.5)$ \\
\hline Erosions, $\mathrm{n}(\%)$ & $16(72.7)$ \\
\hline DAS28, mean (DE) & $2.55(0.7)$ \\
\hline $\mathrm{HAQ}$, mean (DE) & $1.15(0.9)$ \\
\hline \multicolumn{2}{|l|}{ Treatment } \\
\hline sDMARD, n (\%) & $19(86.4)$ \\
\hline Methotrexate, $\mathrm{n}(\%)$ & $8(36.4)$ \\
\hline Leflunomide, n (\%) & $5(22.7)$ \\
\hline Sul fasal azine, n (\%) & $1(4.5)$ \\
\hline Hydr oxychloroquine, $\mathrm{n}(\%)$ & $4(18.2)$ \\
\hline Azathioprine & $1(4.5)$ \\
\hline bDMARDs, n (\%) & $10(45.5)$ \\
\hline Rituximab, $\mathrm{n}(\%)$ & $4(18.2)$ \\
\hline Abatacept, n (\%) & $2(9.1)$ \\
\hline Etanercept, n (\%) & $2(9.1)$ \\
\hline Infliximab, $\mathrm{n}(\%)$ & $1(4.5)$ \\
\hline Adalimumab, n (\%) & $1(4.5)$ \\
\hline
\end{tabular}

Conclusions: Most patients with RA and ILD who are receiving treatment with DMARD $(82 \%)$ remained stable or improved after at least 1 year of both synthetic and biological DMARD treatment. However, a significant percentage of patients had an adverse outcome. More prospective studies with a greater number of patients are necessary to identify the influence of DMARDs in this evolution.

Disclosure of Interest: None declared

DOI: 10.1136/annrheumdis-2017-eular.5824 